\title{
$\mathrm{PH} 104$
}

accualdad

\section{El proyecto europeo SoPHIA reflexiona sobre la calidad de las intervenciones en el patrimonio histórico y cultural}

El proyecto SoPHIA, financiado bajo el programa Horizonte 2020 y liderado por la Universidad Roma Tre, busca proponer un modelo holístico de evaluación de impacto en las intervenciones sobre el patrimonio histórico y cultural, estructurado en torno a tres elementos: las cuatro dimensiones del desarrollo sostenible, los usuarios y el tiempo. Con una duración de dos años, el proyecto se concreta a partir de una primera fase de recopilación y análisis de la literatura, políticas y metodologías, para el diseño de un primer modelo de evaluación de impacto; de una segunda fase de testeo (casos de estudio) para ajustar el modelo; y de una última con propuestas de recomendaciones para el desarrollo de políticas públicas y de programas operativos.

Alberto Cerezo Soto | Fundació Interarts per a la Cooperació Cultural Internacional

URL de la contribución <http://www.iaph.es/revistaph/index.php/revistaph/article/view/5007>

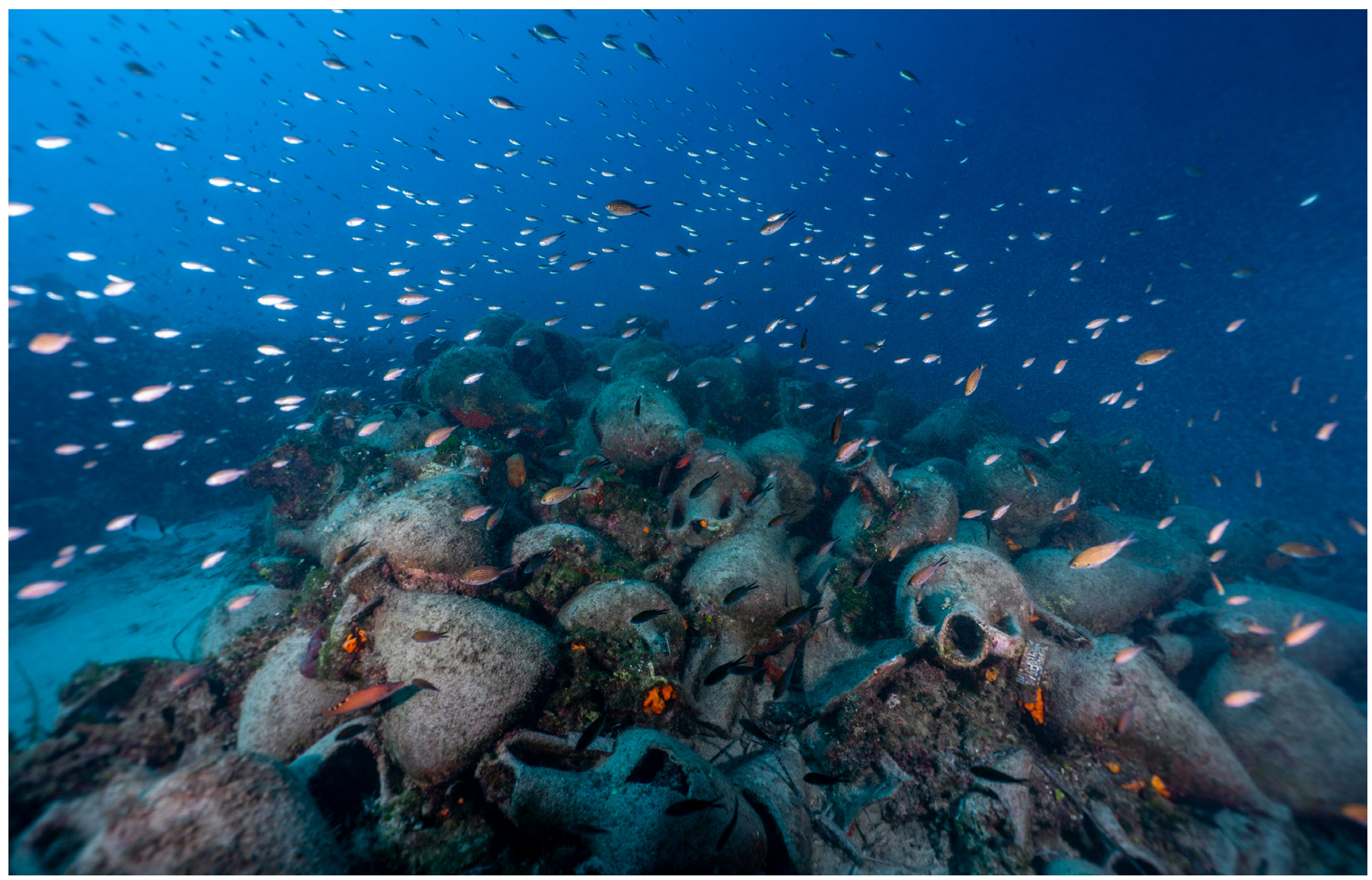

Naufragio en Peristera, Grecia (Ministerio Griego de Cultura y Deportes, Dirección de Antigüedades Subacuáticas), caso de estudio en SoPHIA

| foto Università della Calabria, DIMEG (Matteo Collina) 


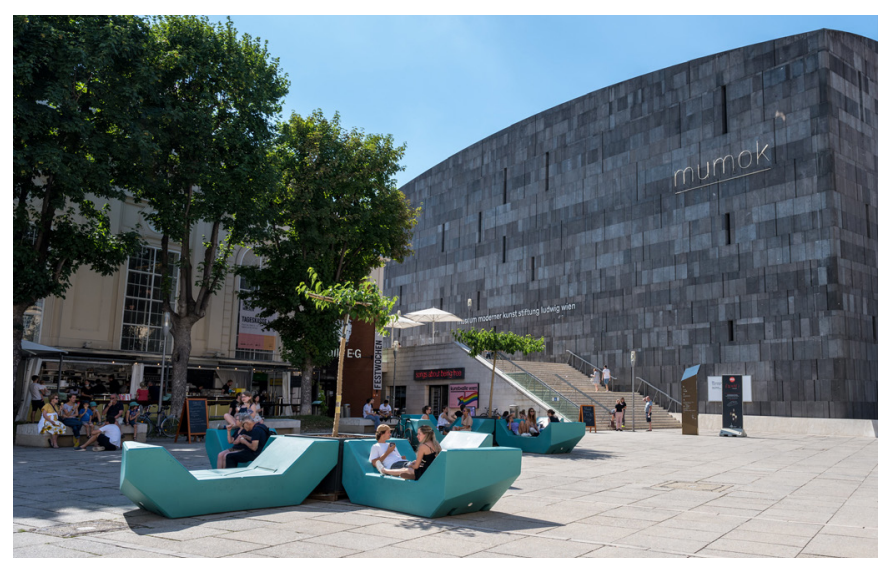

Barrio de los Museos en Viena, caso de estudio SoPHIA | foto Sandor Somkuti

SoPHIA, proyecto desarrollado como plataforma social para la evaluación holística de impacto del patrimonio, está estructurado basándose en tres elementos: las cuatro dimensiones del desarrollo sostenible (economía, sociedad, medio ambiente y cultura); los usuarios (las personas que fruyen del patrimonio); y el tiempo (entendido en su dimensión longitudinal). A lo largo de sus dos años de duración (2020-2021), se concreta a partir de una primera fase de recopilación y análisis de la literatura, políticas y metodologías para el diseño de un primer modelo de evaluación de impacto; de una segunda fase de testeo (casos de estudio) para ajustar el modelo; y de una última con propuestas de recomendaciones para el desarrollo de políticas públicas y de programas operativos. La manera en que el patrimonio cultural es preservado y potenciado es un factor clave en la definición de la identidad europea y su lugar en el mundo. Documentos marco o normativos, tales como el Convenio Europeo del Paisaje (2000) y la Convención de Faro (2005), el Año Europeo del Patrimonio Cultural (2018) y el Marco Europeo de Acción del Patrimonio Cultural (2019), confirman la importancia de evaluar el impacto de las intervenciones en patrimonio cultural, pero también establecen criterios cualitativos y estándares compartidos sobre los que basar dichas evaluaciones. No solo por una importancia relativa al ejercicio mismo sino porque existe un consenso sobre el hecho de que el patrimonio cultural contribuye a muchos de los objetivos de las políticas públicas como la cohesión social, la diversidad y el bienestar individual y colectivo, al crecimiento económico y a la sostenibilidad medioam-

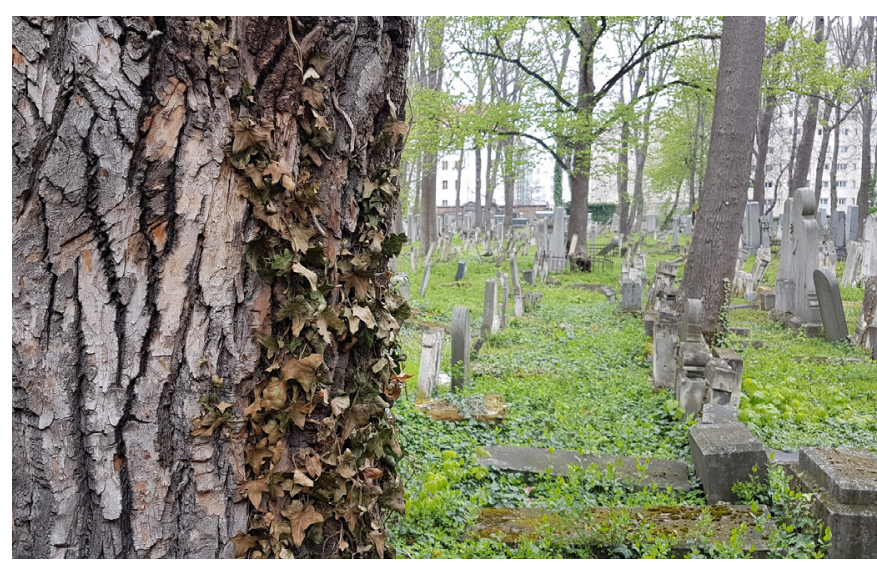

Cementerio Judío Währing, Austria, caso de estudio | foto Monika Bargmann

biental. En otras palabras, el patrimonio cultural ha dejado de considerarse como un ente per se para pasar a ser parte consubstancial de muchas otras dimensiones de las políticas públicas.

Si bien existen algunos ejemplos exitosos de evaluación de impacto, como Impacts 08 de la Capital Europea de la Cultura Liverpool, los actuales modelos de evaluación no proponen un marco exhaustivo que abarque la complejidad de los impactos de las intervenciones sobre el patrimonio cultural. Métodos bien conocidos como la Evaluación de Impacto Ambiental (EIA) o la Evaluación de Impacto del Patrimonio (HIA), a pesar de ser reconocidos y ampliamente aplicados a nivel europeo, no llegan a capturar la naturaleza multidimensional y el alcance de una intervención en patrimonio.

Desde esta perspectiva, SoPHIA ${ }^{1}$ es un proyecto innovador que aborda cuestiones sensibles y de actualidad tanto a nivel europeo como local. De la mencionada revisión inicial de la literatura, resultó un análisis detallado de los métodos de evaluación de impacto actualmente en uso y que sirvió para entender las brechas y oportunidades en los distintos enfoques. Entre otros hallazgos, se identificó la necesidad de emplear metodologías cualitativas y cuantitativas en la evaluación del impacto social del patrimonio cultural y de fortalecer los sistemas de gobernanza para un diálogo constructivo entre la sociedad y las agencias gubernamentales (dimensión social); se ahondó en la relación entre sociedad, individuos y comunidades, y patrimonio para el bienestar 


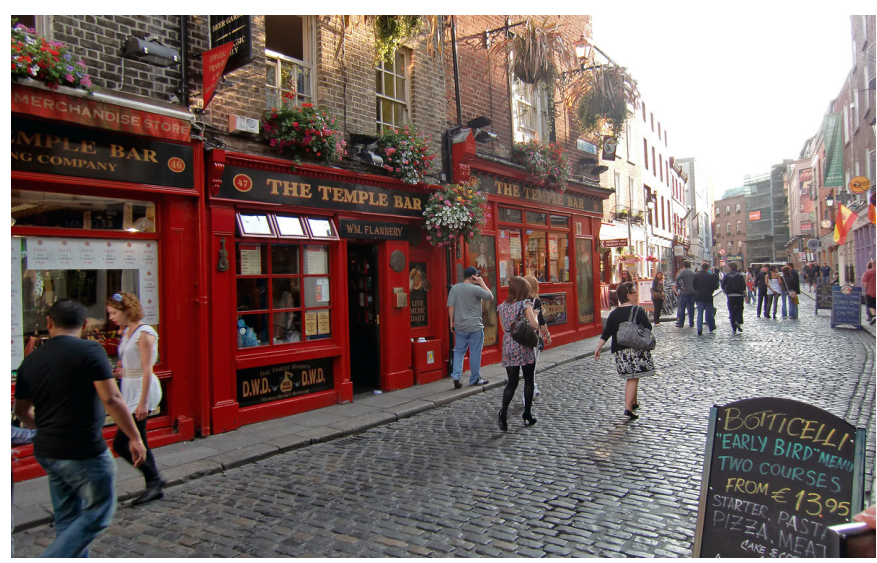

Distrito Cultural Temple Bar (Dublín) caso de estudio SoPHIA | foto Ulrika

y el mantenimiento de la memoria histórica (dimensión cultural); se analizó el concepto de "valor" para entender cuáles de los efectos de las intervenciones tienen connotaciones negativas que suelen ser subestimadas (dimensión económica); se abordó la cuestión de la sostenibilidad, incluyendo temas como el cambio climático, la masificación turística y el crecimiento global urbano (dimensión medioambiental).

Basado en este análisis inicial y en consultas sistemáticas con la comunidad de expertos, se ha diseñado una primera propuesta de modelo de evaluación de impacto que abarca:

$>$ Las conexiones potenciales entre las dimensiones del desarrollo sostenible (económica, social, medioambiental y cultural), así como de las consecuencias no previstas resultado de las intervenciones.

$>$ La involucración de los usuarios (las personas que fruyen del patrimonio) en los procesos de evaluación de impacto, entendiendo que es crucial un ejercicio de fertilización cruzada para una evaluación inclusiva, efectiva y sostenible.

$>$ La perspectiva longitudinal en el tiempo -ex ante, in itinere y ex post- para una gestión y procesos de monitoreo eficientes.

El modelo SoPHIA de evaluación de impacto ha sido testado en doce sitios a lo largo de Europa representativos de la diversidad patrimonial, a saber, un paisaje histórico, dos museos, dos programas, tres distritos culturales, un lugar de memoria, un centro histórico urbano, una isla y un monasterio. De los casos se desprende la complejidad de cada intervención, pero también se apuntan conclusiones interesantes: cómo el patrimonio mejora la calidad de vida de las comunidades; cómo al tiempo contribuye al desarrollo turístico y económico a través de nuevos emprendimientos; y cómo las distintas narrativas que se desprenden contribuyen a dar sentido a una comunidad y fortalecen los procesos de cohesión social.

En este momento, el modelo está siendo ajustado y mejorado a partir de un proceso de consultas. En su última fase, SoPHIA propondrá recomendaciones para el desarrollo de políticas públicas y programas operativos que incluyan las evaluaciones de impacto en las intervenciones sobre el patrimonio cultural desde un enfoque cualitativo.

Como plataforma social, SoPHIA reúne a una comunidad más allá de las instituciones formalmente ligadas al proyecto. Tomadores de decisión, profesionales y académicos son miembros del Consejo Consultivo y del cuerpo de partes interesadas, los stakeholders. Son personas procedentes de toda Europa y vinculadas a instituciones públicas y privadas, universidades y centros de investigación, así como organizaciones de la sociedad civil. Su acercamiento al patrimonio histórico y cultural es muy diverso en términos de especializaciones y enfoques y de misiones y responsabilidades. La comunidad SoPHIA se reúne en los talleres y conferencias del proyecto, son consultados e invitados a discutir en sesiones propuestas regularmente. Además de contribuir activamente al proyecto, la plataforma social se convierte en una herramienta efectiva de diseminación, acercando a sus miembros a la cuestión de la evaluación de impacto

\section{NOTAS}

1. Todos los informes (disponibles en inglés) del proyecto SoPHIA son públicos y pueden ser consultados y descargados desde la sección Archive de la página web del proyecto: https://sophiaplatform.eu/en/archive 